\title{
A LOCAL RESULT ON INSENSITIZING CONTROLS FOR A SEMILINEAR HEAT EQUATION WITH NONLINEAR BOUNDARY FOURIER CONDITIONS*
}

\author{
OLIVIER BODART ${ }^{\dagger}$, MANUEL GONZÁLEZ-BURGOS ${ }^{\ddagger}$, AND ROSARIO PÉREZ-GARCÍA
}

\begin{abstract}
In this paper we present a local result on the existence of insensitizing controls for a semilinear heat equation when nonlinear boundary conditions of the form $\partial_{n} y+f(y)=0$ are considered. The problem leads to an analysis of a special type of nonlinear null controllability problem. A sharp study of the linear case and a later application of an appropriate fixed point argument constitute the scheme of the proof of the main result. The boundary conditions we are dealing with lead us to seek a fixed point, and thus also control functions, in certain Hölder spaces. The main strategy in this paper is the construction of controls with Hölderian regularity starting from $L^{2}$-controls in the linear case. Sufficient regularity in the data and appropriate assumptions on the right-hand side term $\xi$ of the equation are required.
\end{abstract}

Key words. controllability, nonlinear PDE of parabolic type

AMS subject classifications. 93B05, 35K55, 35K05

DOI. $10.1137 /$ S036301290343161X

1. Statement of the problem and main result. Let $\Omega \subset \mathbb{R}^{N}$ be, with $N \geq 1$, a bounded domain with boundary $\partial \Omega$ of at least class $C^{2}$. Let $\omega$ and $\mathcal{O}$ be nonempty open subsets of $\Omega$. For $T>0$, we denote by $Q$ the cylinder $\Omega \times(0, T)$ and by $\Sigma$ its lateral boundary $\partial \Omega \times(0, T)$. We consider a semilinear heat equation with nonlinear boundary conditions of Fourier type and partially known initial data:

$$
\left\{\begin{array}{l}
\partial_{t} y-\Delta y+F(y)=\xi+v \mathbf{1}_{\omega} \quad \text { in } Q \\
\partial_{n} y+f(y)=0 \text { on } \Sigma, \\
y(x, 0)=y_{0}(x)+\tau \hat{y}_{0}(x) \quad \text { in } \Omega
\end{array}\right.
$$

where $F$ and $f$ are given $C^{1}$ functions defined on $\mathbb{R} ; \xi$ and $y_{0}$ are, respectively, a known heat source and a given initial datum, both regular enough; $\tau$ is an unknown small real number; and $\hat{y}_{0}$ is unknown in an appropriate Banach space $X \hookrightarrow L^{2}(\Omega)$ (the embedding being continuous and dense), with $\left\|\hat{y}_{0}\right\|_{X}=1$. Here, $v=v(x, t)$ is a control function to be determined, $\mathbf{1}_{\omega}$ is the characteristic function of the set $\omega$, $\partial_{t}$ denotes the time derivative, and $\partial_{n}$ represents the derivation with respect to the outward unit normal to $\partial \Omega$.

Let us define

$$
\Phi(y)=\frac{1}{2} \iint_{\mathcal{O} \times(0, T)}|y(x, t ; \tau, v)|^{2} d x d t,
$$

$y=y(\cdot, \cdot ; \tau, v)$ being a solution of (1.1) (associated to $\tau$ and $v$ ) defined in $(0, T)$, if one exists. In this paper we analyze the existence of control functions that make

\footnotetext{
${ }^{*}$ Received by the editors July 15, 2003; accepted for publication (in revised form) February 16, 2004; published electronically October 8, 2004. This work has been partially financed by D.G.E.S. (Spain), grant PB98-1134.

http://www.siam.org/journals/sicon/43-3/43161.html

†Laboratoire de Mathématiques Appliquées, UMR CNRS 6620, Université Blaise-Pascal, Clermont-Ferrand 2, 63177 Aubière, France (Olivier.Bodart@math.univ-bpclermont.fr).

${ }^{\ddagger}$ Dpto. Ecuaciones Diferenciales y Análisis Numérico, Universidad de Sevilla, Aptdo. 1160, 41080 Sevilla, Spain (manoloburgos@us.es, rosariopg@us.es).
} 
the functional $\Phi$ locally insensitive to small perturbations in the initial condition. A possible physical interpretation of this problem would be the following. The function $y=y(x, t)$ can be viewed as the relative temperature of a body (with respect to the exterior surrounding air). The semilinear parabolic equation in (1.1) means that there is a fixed heat source $\xi$ acting on the body and that we can also act on a small part $\omega$ of the body by means of a heat source $v \mathbf{1}_{\omega}$. On the boundary, $-\frac{\partial y}{\partial n}$ can be viewed as the normal heat flux, directed inward, up to a positive coefficient. Thus, the equality

$$
-\frac{\partial y}{\partial n}=f(y)
$$

means that this flux is a (nonlinear) function of the temperature. The problem with insensitizing $\Phi$ means that we are seeking a control function acting on $\omega$ such that the energy in $\mathcal{O}$ is invariant for small perturbations in the initial data. A natural physical hypothesis would be to suppose that $f$ is nondecreasing with $f(0)=0$. Throughout this paper, we will assume no special behavior on the increasing of $f$.

By reasons that will be seen later, in this work we will slightly change the usual notion of insensitizing controls (see [1], [4], [10], [11]), which is equivalent to the usual one in the linear case.

Definition 1.1. A control function $v$ is said to insensitize $\Phi$ if there exists $\tau_{0}>0$ such that system (1.1) admits a weak solution $y(\cdot, \cdot ; \tau, v) \in L^{2}\left(0, T ; H^{1}(\Omega)\right) \cap C^{0}(\bar{Q})$ for $|\tau| \leq \tau_{0}$ and if the following insensitivity condition holds:

$$
\left.\frac{\partial \Phi(y(\cdot, \cdot ; \tau, v))}{\partial \tau}\right|_{\tau=0}=0 \quad \forall \hat{y}_{0} \in X \text { with }\left\|\hat{y}_{0}\right\|_{X}=1,
$$

where $X=C^{2+\beta}(\bar{\Omega}) \cap H_{0}^{2}(\Omega)$.

By a weak solution of (1.1) (associated to $\tau$ and $v$ ) we will define a function (if one exists) $y=y(\cdot, \cdot ; \tau, v) \in L^{2}\left(0, T ; H^{1}(\Omega)\right) \cap C^{0}(\bar{Q})$, with $\partial_{t} y \in L^{2}\left(0, T ; H^{-1}(\Omega)\right)$, such that

$$
\left\{\begin{array}{l}
\left\langle\partial_{t} y(t), u\right\rangle_{\left(H^{1}(\Omega)\right)^{\prime}, H^{1}(\Omega)}+\int_{\Omega} \nabla y(t) \cdot \nabla u d x+\int_{\Omega} F(y(t)) u d x+\int_{\partial \Omega} f(y(t)) u d \sigma \\
=\int_{\Omega}\left(\xi(t)+v(t) \mathbf{1}_{\omega}\right) u d x \quad \text { in } L^{2}(0, T) \quad \forall u \in H^{1}(\Omega), \\
y(0)=y_{0}+\tau \hat{y}_{0} .
\end{array}\right.
$$

Insensitivity problems were originally introduced by J.-L. Lions in [10] and were first studied for semilinear heat equations with globally Lipschitz-continuous nonlinearities $F=F(y)$ and Dirichlet boundary conditions. In [1], the existence of the so-called $\varepsilon$-insensitizing controls for partially known data in both the initial and boundary conditions is proved. In [11] it is shown that one cannot expect the existence of insensitizing controls for every $y_{0} \in L^{2}(\Omega)$ when $\Omega \backslash \bar{\omega} \neq \emptyset$, even if $F \equiv 0$. In addition, for $y_{0}=0$ and suitable assumptions on the source term $\xi$, de Teresa proves the existence of insensitizing controls (see Theorem 1 in [11]). This last result is extended in [2] and [3] to nonlinearities with certain superlinear growth at infinity. It is also generalized in [4] to the case of a heat equation with a nonlinear term involving the state $y$ and its gradient. In [4], the authors also present an insensitivity result for a semilinear heat equation with a nonlinear term $F(y)$ and linear boundary conditions of Fourier type. In the present paper, we prove a local result on the existence of insensitizing controls for system (1.1), which is, to our knowledge, the first insensitivity 
result in the literature for a semilinear heat equation with nonlinear Fourier boundary conditions. In the framework of the controllability, both approximate and null controllability of the classical heat equation with nonlinear Fourier boundary conditions are analyzed in [5].

Before stating the main result in this paper, let us introduce the following notation. For $p \in[1, \infty]$ and any Banach space $Y,\|\cdot\|_{L^{p}(Y)}$ will denote the norm in the space $L^{p}(0, T ; Y)$. For simplicity, the norm in $L^{p}(Q)$ will be represented by $\|\cdot\|_{L^{p}}$ for $p \in[1, \infty),\|\cdot\|_{\infty}$ will stand for the norm in $L^{\infty}(Q)$, and $\|\cdot\|_{\infty ; \Sigma}$ will denote the norm in $L^{\infty}(\Sigma)$. For $r \in(2, \infty)$ and any open set $\mathcal{V} \subset \mathbb{R}^{N}$, we introduce the Banach space

$$
X^{r}(0, T ; \mathcal{V})=\left\{u \in L^{r}\left(0, T ; W^{2, r}(\mathcal{V})\right): \partial_{t} u \in L^{r}\left(0, T ; L^{r}(\mathcal{V})\right)\right\},
$$

with its natural norm

$$
\|u\|_{X^{r}(0, T ; \mathcal{V})}=\|u\|_{L^{r}\left(W^{2, r}(\mathcal{V})\right)}+\left\|\partial_{t} u\right\|_{L^{r}\left(L^{r}(\mathcal{V})\right)} .
$$

On the other hand, for $\beta \in(0,1)$ and $u \in C^{0}(\bar{Q})$, we define the quantity

$$
[u]_{\beta, \frac{\beta}{2}}=\sup _{\bar{Q}} \frac{\left|u(x, t)-u\left(x^{\prime}, t\right)\right|}{\left|x-x^{\prime}\right|^{\beta}}+\sup _{\bar{Q}} \frac{\left|u(x, t)-u\left(x, t^{\prime}\right)\right|}{\left|t-t^{\prime}\right|^{\frac{\beta}{2}}} .
$$

We will consider the space $C^{\beta, \frac{\beta}{2}}(\bar{Q})=\left\{u \in C^{0}(\bar{Q}):[u]_{\beta, \frac{\beta}{2}}<\infty\right\}$, which is a Banach space with its natural norm $|u|_{\beta, \frac{\beta}{2} ; \bar{Q}}=\|u\|_{\infty}+[u]_{\beta, \frac{\beta}{2}}$. We will also consider the Banach spaces defined by

$$
\begin{gathered}
C^{1+\beta, \frac{1+\beta}{2}}(\bar{Q})=\left\{u \in C^{0}(\bar{Q}): \frac{\partial u}{\partial x_{i}} \in C^{\beta, \frac{\beta}{2}}(\bar{Q}) \quad \forall i, \sup _{\bar{Q}} \frac{\left|u(x, t)-u\left(x, t^{\prime}\right)\right|}{\left|t-t^{\prime}\right|^{\frac{1+\beta}{2}}}<\infty\right\}, \\
C^{2+\beta, 1+\frac{\beta}{2}}(\bar{Q})=\left\{u \in C^{0}(\bar{Q}): \frac{\partial u}{\partial x_{i}} \in C^{1+\beta, \frac{1+\beta}{2}}(\bar{Q}) \quad \forall i, \partial_{t} u \in C^{\beta, \frac{\beta}{2}}(\bar{Q})\right\},
\end{gathered}
$$

and

$$
C^{3+\beta, \frac{3+\beta}{2}}(\bar{Q})=\left\{u \in C^{0}(\bar{Q}): \frac{\partial u}{\partial x_{i}} \in C^{2+\beta, 1+\frac{\beta}{2}}(\bar{Q}) \quad \forall i, \partial_{t} u \in C^{1+\beta, \frac{1+\beta}{2}}(\bar{Q})\right\},
$$

with norms denoted by $|\cdot|_{n+\beta, \frac{n+\beta}{2} ; \bar{Q}}, n=1,2,3$. The Banach space formed by the restrictions to $\bar{\Sigma}$ of the functions in $C^{n+\beta, \frac{n+\beta}{2}}(\bar{Q})$ will be represented by $C^{n+\beta, \frac{n+\beta}{2}}(\bar{\Sigma})$ and its norm by $|\cdot|_{n+\beta, \frac{n+\beta}{2} ; \bar{\Sigma}}$. Finally, we shall write $|\cdot|_{2+\beta ; \bar{\Omega}}$ to denote the norm in $C^{2+\beta}(\bar{\Omega})$, and the norm in the space $L^{2}\left(0, T ; H^{1}(\Omega)\right) \cap C\left([0, T] ; L^{2}(\Omega)\right)$ will be denoted by $\|\cdot\|_{L^{2}\left(H^{1}\right) \cap C\left(L^{2}\right)}$.

The main goal in this paper is to prove the following local insensitivity result for system (1.1).

TheOrem 1.2. Assume that $\partial \Omega \in C^{3+\beta}$ for some $\beta \in(0,1), \omega \cap \mathcal{O} \neq \emptyset$, and $y_{0}=0$. Let $F, f \in C^{3}(\mathbb{R})$ verify $F(0)=f(0)=0$. Then, there exist two positive constants $\mathcal{M}$ and $\eta$ (depending on $\Omega, \omega, \mathcal{O}, T, F$, and $f$ ) such that, for any $\xi \in C^{\beta, \frac{\beta}{2}}(\bar{Q})$ satisfying

$$
|\xi|_{\beta, \frac{\beta}{2} ; \bar{Q}}+\left\|\exp \left(\frac{\mathcal{M}}{2 t}\right) \xi\right\|_{L^{2}} \leq \eta
$$


one can find a control function $v \in C^{\beta, \frac{\beta}{2}}(\bar{Q})$ that insensitizes the functional $\Phi$ defined by (1.2).

It is of interest to notice that the explicit way the constant $\mathcal{M}$ depends on $T$ and $F$ can be known (see Remark 1).

As usual in insensitivity problems, the insensitivity condition (1.3) leads us to analyze a nonstandard nonlinear null controllability problem. In the case under consideration, the following holds.

Proposition 1.3. If there exists a control function $v$ insensitizing the functional $\Phi$ given by (1.2), then this control $v$ solves the null controllability problem

$$
\begin{gathered}
\left\{\begin{array}{l}
\partial_{t} \bar{y}-\Delta \bar{y}+F(\bar{y})=\xi+v \mathbf{1}_{\omega} \quad \text { in } Q, \\
\partial_{n} \bar{y}+f(\bar{y})=0 \quad \text { on } \Sigma, \\
\bar{y}(x, 0)=y_{0}(x) \quad \text { in } \Omega,
\end{array}\right. \\
\left\{\begin{array}{l}
-\partial_{t} q-\Delta q+F^{\prime}(\bar{y}) q=\bar{y} \mathbf{1}_{\mathcal{O}} \quad \text { in } Q, \\
\partial_{n} q+f^{\prime}(\bar{y}) q=0 \quad \text { on } \Sigma, \\
q(x, T)=0 \quad \text { in } \Omega,
\end{array}\right. \\
q(x, 0)=0 \quad \text { in } \Omega .
\end{gathered}
$$

Furthermore, if a control function $v$ solves (1.5)-(1.7) and there exists $\tau_{0}>0$ such that (1.1) admits a weak solution $y(\cdot, \cdot ; \tau, v) \in L^{2}\left(0, T ; H^{1}(\Omega)\right) \cap C^{0}(\bar{Q})$ for $|\tau| \leq \tau_{0}$, then $v$ is insensitizing the functional $\Phi$.

Proof. We reason as in [10] and [1]. Assume the existence of a control $v$ insensitizing the functional $\Phi$ given by (1.2) in the sense of Definition 1.1. Then, system (1.5) admits a weak solution $y(\cdot, \cdot ; \tau, v) \in L^{2}\left(0, T ; H^{1}(\Omega)\right) \cap C^{0}(\bar{Q})$ for all $|\tau| \leq \tau_{0}$, for some $\tau_{0}>0$. The derivative of $\Phi(y(\cdot, \cdot ; \tau, v))$ with respect to $\tau$ at $\tau=0$ is given by

$$
\left.\frac{\partial \Phi(y(\cdot, \cdot ; \tau, v))}{\partial \tau}\right|_{\tau=0}=\iint_{Q} \bar{y}(x, t) \mathbf{1}_{\mathcal{O}} y_{\tau}(x, t) d x d t
$$

where $\bar{y}=y(\cdot, \cdot ; 0, v) \in C^{0}(\bar{Q})$ and $y_{\tau}=\left.\frac{\partial y(\cdot, \cdot ; \tau, v)}{\partial \tau}\right|_{\tau=0}$ is the solution of the linear system

$$
\left\{\begin{array}{l}
\partial_{t} y_{\tau}-\Delta y_{\tau}+F^{\prime}(\bar{y}) y_{\tau}=0 \quad \text { in } Q \\
\partial_{n} y_{\tau}+f^{\prime}(\bar{y}) y_{\tau}=0 \quad \text { on } \Sigma \\
y_{\tau}(x, 0)=\hat{y}_{0}(x) \text { in } \Omega .
\end{array}\right.
$$

Let $q$ be the solution of (1.6). Replacing $\bar{y} \mathbf{1}_{\mathcal{O}}$ with the left-hand side of the PDE satisfied by $q$ and integrating by parts, one obtains

$$
\iint_{Q} \bar{y}(x, t) \mathbf{1}_{\mathcal{O}} y_{\tau}(x, t) d x d t=\int_{\Omega} q(x, 0) \hat{y}_{0}(x) d x
$$

regardless of what $\hat{y}_{0} \in L^{2}(\Omega)$ is. Finally, from (1.3) one deduces that

$$
q(0)=0 \quad \text { in } X^{\prime},
$$


whence (1.7) follows, in view of the Hahn-Banach theorem. The rest of the proof follows immediately from Definition 1.1.

Notice that a control function $v$ solving (1.5)-(1.7), if one exists, does not necessarily insensitize the functional $\Phi$ (think, for instance, of an initial datum $y_{0}+\tau \hat{y}_{0}$ not lying in $C^{0}(\bar{\Omega})$, for which system (1.1) admits no weak solution in $C^{0}(\bar{Q})$ ). In other words, in this case the problem of seeking insensitizing controls cannot be reformulated in an equivalent way as a null controllability problem, as is usual in insensitivity problems. In order to prove Theorem 1.2, we will thus argue as follows (see section 3). Under the assumptions in the theorem, we will first prove the existence of a control $v$ solving (1.5)-(1.7). In a second step, we will see that, for $\tau \hat{y}_{0}$ regular and small enough, such a control $v$ can be chosen so that it also insensitizes the functional $\Phi$ defined by (1.2).

The existence of a control function solving (1.5)-(1.7) will be proved by linearization and a later application of an appropriate fixed point argument. This technique, introduced in [12] in the context of the controllability of the semilinear wave equation, has been used to prove several controllability results (cf., for example, [6], [7]). Analyzing a linear null controllability problem similar to (1.5)-(1.7) (see (2.1), (2.2), and (1.7)), we realize that the potentials $a, b \in L^{\infty}(\Sigma)$ need to have time derivatives in $L^{\infty}(\Sigma)$. This requirement comes from applying Lemma 1.2 of [8] to obtain an adequate observability inequality (see Proposition 2.1) for the solutions of the corresponding adjoint systems (2.5) and (2.6). To solve the nonlinear problem, we would have to search for a fixed point in a space containing the functions $z \in L^{\infty}(Q)$ such that the trace of $\partial_{t} z$ lies in $L^{\infty}(\Sigma)$. As was observed in Remark 15 of [5], we are not too far from imposing $\partial_{t} z \in L^{\infty}\left(0, T ; W^{1, N+\gamma}(\Omega)\right)$, with $\gamma>0$. But these spaces are too small to achieve compactness and good estimates for the fixed point mapping. We will then seek a fixed point, and thus also control functions, in the Hölder spaces introduced above. In fact, one of the main points in this paper relies on the construction, in the linear case, of control functions with Hölderian regularity starting from $L^{2}$-controls.

In order to ensure the existence of a solution to system (1.1) in the abovementioned Hölder spaces, appropriate regularity assumptions on the data and a compatibility condition on the initial datum are required (see Lemma 3.2). This is the reason why we have introduced the space $X=C^{2+\beta}(\bar{\Omega}) \cap H_{0}^{2}(\Omega)$, with $\beta \in(0,1)$, in Definition 1.1.

In the next section, we will analyze the corresponding linear null controllability problem, while section 3 will be devoted to proving our main result.

2. The linear null controllability problem. From now on, we will assume that $\omega \cap \mathcal{O} \neq \emptyset$ and $y_{0}=0$. This section is devoted to solving a linearized version of the null controllability problem (1.5)-(1.7). We consider the linear systems

$$
\begin{aligned}
& \left\{\begin{array}{l}
\partial_{t} y-\Delta y+c y=\xi+v \mathbf{1}_{\omega} \text { in } Q, \\
\partial_{n} y+a y=0 \text { on } \Sigma \\
y(x, 0)=0 \text { in } \Omega
\end{array}\right. \\
& \left\{\begin{array}{l}
-\partial_{t} q-\Delta q+d q=y \mathbf{1}_{\mathcal{O}} \text { in } Q, \\
\partial_{n} q+b q=0 \text { on } \Sigma \\
q(x, T)=0 \text { in } \Omega
\end{array}\right.
\end{aligned}
$$


where $a, b \in L^{\infty}(\Sigma), c, d \in L^{\infty}(Q)$, and $\xi \in L^{2}(Q)$ (at least). For each $v \in L^{2}(Q)$, the cascade of linear systems $(2.1),(2.2)$ admits exactly one solution $(y, q)$ satisfying

$$
y, q \in L^{2}\left(0, T ; H^{1}(\Omega)\right) \cap C\left([0, T] ; L^{2}(\Omega)\right), \quad \partial_{t} y, \partial_{t} q \in L^{2}\left(0, T ; H^{-1}(\Omega)\right),
$$

with

$$
\begin{aligned}
& \|y\|_{L^{2}\left(H^{1}\right) \cap C\left(L^{2}\right)}+\left\|\partial_{t} y\right\|_{L^{2}\left(H^{-1}\right)} \leq C\left(\Omega, T,\|a\|_{\infty ; \Sigma},\|c\|_{\infty}\right)\left(\|\xi\|_{L^{2}}+\|v\|_{L^{2}}\right) \\
& \|q\|_{L^{2}\left(H^{1}\right) \cap C\left(L^{2}\right)}+\left\|\partial_{t} q\right\|_{L^{2}\left(H^{-1}\right)} \\
& \quad \leq C\left(\Omega, T,\|a\|_{\infty ; \Sigma},\|b\|_{\infty ; \Sigma},\|c\|_{\infty},\|d\|_{\infty}\right)\left(\|\xi\|_{L^{2}}+\|v\|_{L^{2}}\right) .
\end{aligned}
$$

Under additional assumptions on the potentials and on the source term $\xi$, we will build a regular control $v$, acting on a nonempty open subset of $\omega \cap \mathcal{O}$, such that the corresponding solution $(y, q)$ of $(2.1),(2.2)$ satisfies $(1.7)$.

We proceed as follows. Let us fix a nonempty open set $B_{0}$ such that $B_{0} \subset \subset \omega \cap \mathcal{O}$. In a first step, using an appropriate observability inequality, we obtain an $L^{2}$-control supported on $\bar{B}_{0} \times[0, T]$. Then, by means of a construction similar to that made in [2] and [3] and due to the regularizing properties of the heat equation, we will be able to furnish a regular control with a slightly larger support.

Let us consider the adjoint systems

$$
\begin{aligned}
& \left\{\begin{array}{l}
\partial_{t} \varphi-\Delta \varphi+d \varphi=0 \quad \text { in } Q \\
\partial_{n} \varphi+b \varphi=0 \text { on } \Sigma \\
\varphi(x, 0)=\varphi^{0}(x) \text { in } \Omega
\end{array}\right. \\
& \left\{\begin{array}{l}
-\partial_{t} \psi-\Delta \psi+c \psi=\varphi \mathbf{1}_{\mathcal{O}} \text { in } Q, \\
\partial_{n} \psi+a \psi=0 \text { on } \Sigma, \\
\psi(x, T)=0 \text { in } \Omega,
\end{array}\right.
\end{aligned}
$$

where $\varphi^{0} \in L^{2}(\Omega)$. For simplicity, we will denote by $a_{t}$ (resp., $b_{t}$ ) the time derivative of $a$ (resp., of $b$ ). Let $B_{0} \subset \subset \omega \cap \mathcal{O}$ be the open set considered above. In [4], the following observability inequality for the solutions of (2.5), (2.6) is proved.

Proposition 2.1. Assume that $a, b, a_{t}, b_{t} \in L^{\infty}(\Sigma)$ and $c, d \in L^{\infty}(Q)$. Then, there exist positive constants $M$ and $C$, depending on $\Omega, \omega, \mathcal{O}, T,\|a\|_{\infty ; \Sigma},\|b\|_{\infty ; \Sigma}$, $\left\|a_{t}\right\|_{\infty ; \Sigma},\left\|b_{t}\right\|_{\infty ; \Sigma},\|c\|_{\infty}$, and $\|d\|_{\infty}$, such that

$$
\iint_{Q} \exp \left(-\frac{M}{t}\right)|\psi|^{2} d x d t \leq C \iint_{B_{0} \times(0, T)}|\psi|^{2} d x d t
$$

for every $\varphi^{0} \in L^{2}(\Omega)$, where $\psi$ solves (2.6), $\varphi$ being the solution of (2.5).

The proof of this result follows the scheme of demonstration of Proposition 2 in [11] and uses a global Carleman inequality for the classical heat equation with linear boundary Fourier conditions (see Lemma 1.2 of [8]).

Remark 1. In the previous proposition, and throughout this section, the dependence of the constants with respect to $T$ and the potentials $c$ and $d$ could be stated precisely. This would allow one to know the precise way the constant $\mathcal{M}$ in Theorem 1.2 depends on $T$ and $F$. Nevertheless, the dependence on the boundary data $a$ and $b$ is not explicit. This comes from the proof of Lemma 1.2 of [8]. 
Due to a unique continuation property for the solutions of (2.5) and (2.6) inferred from Proposition 2.1, under suitable assumptions on $\xi$, one obtains $L^{2}$-controls as follows.

Proposition 2.2. Assume that $a, b, a_{t}, b_{t} \in L^{\infty}(\Sigma)$ and $c, d \in L^{\infty}(Q)$. Let $M$ and $C$ be the positive constants (depending on $\Omega, \omega, \mathcal{O}, T,\|a\|_{\infty ; \Sigma},\|b\|_{\infty ; \Sigma},\left\|a_{t}\right\|_{\infty ; \Sigma}$, $\left\|b_{t}\right\|_{\infty ; \Sigma},\|c\|_{\infty}$, and $\left.\|d\|_{\infty}\right)$ provided by Proposition 2.1. Then, for any $\xi \in L^{2}(Q)$ verifying

$$
\iint_{Q} \exp \left(\frac{M}{t}\right)|\xi|^{2} d x d t<\infty
$$

there exists a control function $\hat{v} \in L^{2}(Q)$, with supp $\hat{v} \subset \bar{B}_{0} \times[0, T]$, such that the solution $(\hat{y}, \hat{q})$ of (2.1), (2.2) associated to $\hat{v}$ satisfies (1.7). Moreover, $\hat{v}$ can be chosen so that

$$
\|\hat{v}\|_{L^{2}} \leq \sqrt{C}\left(\iint_{Q} \exp \left(\frac{M}{t}\right)|\xi|^{2} d x d t\right)^{1 / 2}
$$

The proof of this proposition is given in [4] and will be omitted here. The main result in this section is the following.

Proposition 2.3. Assume that $\partial \Omega \in C^{3+\beta}$ for some $\beta \in(0,1), a, b, a_{t}, b_{t} \in$ $L^{\infty}(\Sigma), c \in C^{\beta, \frac{\beta}{2}}(\bar{Q})$, and $d \in C^{1+\beta, \frac{1+\beta}{2}}(\bar{Q})$. Let $M>0$ be the constant (depending on $\Omega, \omega, \mathcal{O}, T,\|a\|_{\infty ; \Sigma},\|b\|_{\infty ; \Sigma},\left\|a_{t}\right\|_{\infty ; \Sigma},\left\|b_{t}\right\|_{\infty ; \Sigma},\|c\|_{\infty}$, and $\left.\|d\|_{\infty}\right)$ provided by Proposition 2.1. Then, for any $\xi \in C^{\beta, \frac{\beta}{2}}(\bar{Q})$ satisfying (2.7), one can find a control $v \in C^{\beta, \frac{\beta}{2}}(\bar{Q})$ such that the associated solution $(y, q)$ of $(2.1)$, (2.2) satisfies (1.7). Moreover, the $C^{\beta, \frac{\beta}{2}}$-norm of $v$ can be estimated as follows:

$$
|v|_{\beta, \frac{\beta}{2} ; \bar{Q}} \leq C\left(|\xi|_{\beta, \frac{\beta}{2} ; \bar{Q}}+\left\|\exp \left(\frac{M}{2 t}\right) \xi\right\|_{L^{2}}\right)
$$

where $C$ is a new positive constant depending on $\Omega, \omega, \mathcal{O}, T,\|a\|_{\infty ; \Sigma},\|b\|_{\infty ; \Sigma}$, $\left\|a_{t}\right\|_{\infty ; \Sigma},\left\|b_{t}\right\|_{\infty ; \Sigma},|c|_{\beta, \frac{\beta}{2} ; \bar{Q}}$, and $|d|_{1+\beta, \frac{1+\beta}{2} ; \bar{Q}}$.

The regularity of $v$ and, accordingly, that of $(y, q)$, will enable us to deal with the nonlinear null controllability problem (1.5)-(1.7).

Before proving Proposition 2.3, for the convenience of the reader we repeat some relevant material from [3] and [9] without proofs, thus making our exposition selfcontained. We first recall a technical result on local regularity given in [3, Proposition 2.1 and Remark 4].

LEMma 2.4. Let $\widetilde{a} \in L^{\infty}(Q)$ and $h \in L^{2}(Q)$ be given. Let us consider a solution $u \in L^{2}\left(0, T ; H^{1}(\Omega)\right) \cap C\left([0, T] ; L^{2}(\Omega)\right)$ of

$$
\left\{\begin{array}{l}
\partial_{t} u-\Delta u+\widetilde{a} u=h \quad \text { in } Q, \\
u(x, 0)=0 \quad \text { in } \Omega,
\end{array}\right.
$$

and let $\mathcal{V} \subset \Omega$ be an arbitrary open set.

(a) If $h \in L^{r}\left(0, T ; L^{r}(\mathcal{V})\right)$, with $r \in(2, \infty)$, then $u \in X^{r}\left(0, T ; \mathcal{V}^{\prime}\right)$ for any open set $\mathcal{V}^{\prime} \subset \subset \mathcal{V}$. Moreover, there exist two positive constants $C=C\left(\Omega, \mathcal{V}, \mathcal{V}^{\prime}, T, N, r\right)$ and $\mathcal{K}=\mathcal{K}(N)$ such that

$$
\|u\|_{X^{r}\left(0, T ; \mathcal{V}^{\prime}\right)} \leq C\left(1+\|\widetilde{a}\|_{\infty}\right)^{\mathcal{K}}\left[\|h\|_{L^{r}\left(L^{r}(\mathcal{V})\right)}+\|u\|_{L^{2}\left(H^{1}\right) \cap C\left(L^{2}\right)}\right] .
$$



with

(b) Assume, in addition, that $h \in L^{r}\left(0, T ; W^{1, r}(\mathcal{V})\right), r>2$, and $\nabla \widetilde{a} \in L^{\gamma}(Q)^{N}$,

$$
\gamma=\left\{\begin{array}{cl}
\max \left\{r, \frac{N}{2}+1\right\} & \text { if } \quad r \neq \frac{N}{2}+1, \\
\frac{N}{2}+1+\varepsilon & \text { if } \quad r=\frac{N}{2}+1,
\end{array}\right.
$$

and $\varepsilon$ being an arbitrarily small positive number. Then, for any open set $\mathcal{V}^{\prime} \subset \subset \mathcal{V}$, one has $u \in L^{r}\left(0, T ; W^{3, r}\left(\mathcal{V}^{\prime}\right)\right), \partial_{t} u \in L^{r}\left(0, T ; W^{1, r}\left(\mathcal{V}^{\prime}\right)\right)$, and for a new positive constant $C=C\left(\Omega, \mathcal{V}, \mathcal{V}^{\prime}, T, N, r\right)$, the following estimate holds:

$$
\|u\|_{L^{r}\left(W^{3, r}\left(\mathcal{V}^{\prime}\right)\right)}+\left\|\partial_{t} u\right\|_{L^{r}\left(W^{1, r}\left(\mathcal{V}^{\prime}\right)\right)} \leq C \mathcal{H}\left[\|h\|_{L^{r}\left(W^{1, r}(\mathcal{V})\right)}+\|u\|_{L^{2}\left(H^{1}\right) \cap C\left(L^{2}\right)}\right],
$$

where

$$
\mathcal{H}=\mathcal{H}\left(N,\|\widetilde{a}\|_{\infty},\|\nabla \widetilde{a}\|_{L^{\gamma}}\right)=\left(1+\|\widetilde{a}\|_{\infty}\right)^{\mathcal{K}+1}\left(1+\|\nabla \widetilde{a}\|_{L^{\gamma}}\right),
$$

$\mathcal{K}=\mathcal{K}(N)$ being as in (2.11).

We also recall the following result, which is readily obtained by rewriting Lemma 3.3 of $[9$, p. 80$]$ with the notation introduced at the beginning of this paper (also see Lemma 2.3 of [3]).

Lemma 2.5. Let $\mathcal{V} \subset \mathbb{R}^{N}, N \geq 1$, be a regular open set. The following continuous embeddings hold:

1. If $r<\frac{N}{2}+1$, then $X^{r}(0, T ; \mathcal{V}) \hookrightarrow L^{p}(\mathcal{V} \times(0, T))$, where $\frac{1}{p}=\frac{1}{r}-\frac{2}{N+2}$.

2. If $r=\frac{N}{2}+1$, then $X^{r}(0, T ; \mathcal{V}) \hookrightarrow L^{q}(\mathcal{V} \times(0, T))$ for all $q<\infty$.

3. If $\frac{N}{2}+1<r<N+2$, then $X^{r}(0, T ; \mathcal{V}) \hookrightarrow C^{\alpha, \frac{\alpha}{2}}(\overline{\mathcal{V}} \times[0, T]), \alpha=2-\frac{N+2}{r}$.

4. If $r=N+2$, then $X^{r}(0, T ; \mathcal{V}) \hookrightarrow C^{l, \frac{l}{2}}(\overline{\mathcal{V}} \times[0, T])$ for all $l \in(0,1)$.

5. If $r>N+2$, then $X^{r}(0, T ; \mathcal{V}) \hookrightarrow C^{1+\beta, \frac{1+\beta}{2}}(\overline{\mathcal{V}} \times[0, T])$, where $\beta=1-\frac{N+2}{r}$.

We are now ready to prove Proposition 2.3. From now on, we will specify only the dependence of the constants on the arguments that will be relevant in our analysis. Thus, for instance, the dependence on the dimension $N$, on $B_{0}$, or on the other open sets appearing later will be omitted.

Proof of Proposition 2.3. Assume that $\partial \Omega \in C^{3+\beta}$ for some $\beta \in(0,1)$. Let $a$, $b, c$, and $d$ be as in the statement, and let $M>0$ be provided by Proposition 2.1. Given $\xi \in C^{\beta, \frac{\beta}{2}}(\bar{Q})$ verifying (2.7), Proposition 2.2 provides a control $\hat{v} \in L^{2}(Q)$ such that the associated solution $(\hat{y}, \hat{q})$ of $(2.1),(2.2)$ satisfies (1.7). Moreover, $\hat{v}$ verifies estimate (2.8) and $\operatorname{supp} \hat{v} \subset \bar{B}_{0} \times[0, T]$, with $B_{0}$ being the open set considered at the beginning of this section. One has $\hat{y}, \hat{q} \in L^{2}\left(0, T ; H^{1}(\Omega)\right) \cap C\left([0, T] ; L^{2}(\Omega)\right)$, $\partial_{t} \hat{y}, \partial_{t} \hat{q} \in L^{2}\left(0, T ; H^{-1}(\Omega)\right)$, and estimates such as (2.3) and (2.4) hold.

Let $B, B_{1}$, and $B_{2}$ be regular open sets such that $B_{0} \subset \subset B_{1} \subset \subset B_{2} \subset \subset B \subset \subset$ $\omega \cap \mathcal{O}$. As was anticipated above, a construction similar to the one made in [2] and [3] will allow one to construct a regular control supported on $\bar{B} \times[0, T]$. Indeed, we set

$$
\begin{gathered}
q=(1-\theta) \hat{q}, \\
y=(1-\theta) \hat{y}+2 \nabla \theta \cdot \nabla \hat{q}+(\Delta \theta) \hat{q},
\end{gathered}
$$

with $\theta \in \mathcal{D}(B)$ satisfying $\theta \equiv 1$ in $B_{2}$. We will analyze the interior regularity of $\hat{y}$ and $\hat{q}$, inferring that $(y, q)$ solves $(2.1),(2.2)$, and (1.7) with control term $v \in C^{\beta, \frac{\beta}{2}}(Q)$ given by

$$
v=-\theta \xi+2 \nabla \theta \cdot \nabla \hat{y}+(\Delta \theta) \hat{y}+\left(\partial_{t}-\Delta+c\right)[2 \nabla \theta \cdot \nabla \hat{q}+(\Delta \theta) \hat{q}],
$$


which is, in fact, supported on $B \times[0, T]$.

First, as $c \in L^{\infty}(Q)$ and $\xi+\hat{v} \mathbf{1}_{B_{0}} \in L^{2}(Q) \cap L^{\infty}\left(0, T ; L^{\infty}\left(\Omega \backslash \bar{B}_{0}\right)\right)$, one can apply Lemma 2.4 with $r=(N+2) /(1-\beta), \beta \in(0,1)$ given in the statement, to deduce that $\hat{y}$ lies in $X^{r}\left(0, T ;(\omega \cap \mathcal{O}) \backslash \bar{B}_{1}\right)$ (notice that, without loss of generality, we can assume that $\omega \cap \mathcal{O} \subset \subset \Omega$ and that $\omega \cap \mathcal{O}$ is regular enough). Since $r>N+2$, this space is continuously embedded in $C^{1+\beta, \frac{1+\beta}{2}}\left(\overline{(\omega \cap \mathcal{O}) \backslash B_{1}} \times[0, T]\right)$, by Lemma 2.5 . Thus,

$$
\hat{y} \in C^{1+\beta, \frac{1+\beta}{2}}\left(\overline{(\omega \cap \mathcal{O}) \backslash B_{1}} \times[0, T]\right),
$$

and estimates (2.11) and (2.3) give

$$
|\hat{y}|_{1+\beta, \frac{1+\beta}{2} ; \overline{(\omega \cap \mathcal{O}) \backslash B_{1}} \times[0, T]} \leq C\left(\Omega, \omega, \mathcal{O}, T,\|a\|_{\infty ; \Sigma},\|c\|_{\infty}\right)\left(\|\xi\|_{\infty}+\|\hat{v}\|_{L^{2}}\right) .
$$

By the choice of $\theta$, the term $v_{1}=2 \nabla \theta \cdot \nabla \hat{y}+(\Delta \theta) \hat{y}$ in $(2.14)$ then lies in $C^{\beta, \frac{\beta}{2}}(\bar{Q})$ and one can estimate

$$
\left|v_{1}\right|_{\beta, \frac{\beta}{2} ; \bar{Q}} \leq C\left(\Omega, \omega, \mathcal{O}, T,\|a\|_{\infty ; \Sigma},\|c\|_{\infty}\right)\left(\|\xi\|_{\infty}+\|\hat{v}\|_{L^{2}}\right)
$$

According to the interior regularity of $\hat{y}$, an argument such as the one above implies that $\hat{q} \in C^{1+\beta, \frac{1+\beta}{2}}\left(\overline{B \backslash B_{2}} \times[0, T]\right)$, and estimates (2.11), (2.16), and (2.4) give

$$
|\hat{q}|_{1+\beta, \frac{1+\beta}{2} ; \overline{B \backslash B_{2}} \times[0, T]} \leq C\left(\|\xi\|_{\infty}+\|\hat{v}\|_{L^{2}}\right)
$$

with $C=C\left(\Omega, \omega, \mathcal{O}, T,\|a\|_{\infty ; \Sigma},\|b\|_{\infty ; \Sigma},\|c\|_{\infty},\|d\|_{\infty}\right)$. By the $C^{\beta, \frac{\beta}{2}}$-regularity of $\xi$ and $c$, it is then clear that $v_{2}=-\theta \xi+c[2 \nabla \theta \cdot \nabla \hat{q}+(\Delta \theta) \hat{q}] \in C^{\beta, \frac{\beta}{2}}(\bar{Q})$ and

$$
\left|v_{2}\right|_{\beta, \frac{\beta}{2} ; \bar{Q}} \leq C(\Omega, \omega, \mathcal{O})\left(|\xi|_{\beta, \frac{\beta}{2} ; \bar{Q}}+|c|_{\beta, \frac{\beta}{2} ; \bar{Q}}|\hat{q}|_{1+\beta, \frac{1+\beta}{2} ; \overline{B \backslash B_{2}} \times[0, T]}\right)
$$

which combined with (2.17), yields

$$
\left|v_{2}\right|_{\beta, \frac{\beta}{2} ; \bar{Q}} \leq C\left(\Omega, \omega, \mathcal{O}, T,\|a\|_{\infty ; \Sigma},\|b\|_{\infty ; \Sigma},|c|_{\beta, \frac{\beta}{2} ; \bar{Q}},\|d\|_{\infty}\right)\left(|\xi|_{\beta, \frac{\beta}{2} ; \bar{Q}}+\|\hat{v}\|_{L^{2}}\right) .
$$

We now analyze the term $v_{3}=\left(\partial_{t}-\Delta\right)[2 \nabla \theta \cdot \nabla \hat{q}+(\Delta \theta) \hat{q}]$. To this end, we use the following result on interior Hölderian regularity, whose proof is given at the end of this section.

Lemma 2.6. Assume that $\partial \Omega \in C^{3+\beta}$ for some $\beta \in(0,1)$. Let us consider a solution $u \in L^{2}\left(0, T ; H^{1}(\Omega)\right) \cap C\left([0, T] ; L^{2}(\Omega)\right)$ of $(2.10)$, with $\widetilde{a} \in C^{1+\beta, \frac{1+\beta}{2}}(\bar{Q})$ and $h \in L^{2}(Q) \cap C^{1+\beta, \frac{1+\beta}{2}}(\overline{\mathcal{V}} \times[0, T]), \mathcal{V}$ being a nonempty open subset of $\Omega$. Then, for any open set $\mathcal{V}^{\prime} \subset \subset \mathcal{V}$, one has $u \in C^{3+\beta, \frac{3+\beta}{2}}\left(\overline{\mathcal{V}}^{\prime} \times[0, T]\right)$ and

$$
|u|_{3+\beta, \frac{3+\beta}{2} ; \overline{\mathcal{V}}^{\prime} \times[0, T]} \leq C\left(|h|_{1+\beta, \frac{1+\beta}{2} ; \overline{\mathcal{V}} \times[0, T]}+\|u\|_{L^{2}\left(H^{1}\right) \cap C\left(L^{2}\right)}\right),
$$

where $C$ is a positive constant depending on $\Omega, \mathcal{V}, \mathcal{V}^{\prime}, T$, and $|\widetilde{a}|_{1+\beta, \frac{1+\beta}{2} ; \bar{Q}}$. 
On account of (2.15) and the regularity of the potential $d$, Lemma 2.6 can be applied to $u=\hat{q}$, with $\mathcal{V}=(\omega \cap \mathcal{O}) \backslash \bar{B}_{1}, \mathcal{V}^{\prime}=B \backslash \bar{B}_{2}, h=\hat{y} \mathbf{1}_{\mathcal{O}}$, and $\widetilde{a}=d$, to deduce that $\hat{q}$ lies in $C^{3+\beta, \frac{3+\beta}{2}}\left(\overline{B \backslash B_{2}} \times[0, T]\right)$. Moreover, estimates (2.18), (2.16), and (2.4) give

$$
|\hat{q}|_{3+\beta, \frac{3+\beta}{2} ; \overline{B \backslash B_{2}} \times[0, T]} \leq C\left(\|\xi\|_{\infty}+\|\hat{v}\|_{L^{2}}\right),
$$

with $C=C\left(\Omega, \omega, \mathcal{O}, T,\|a\|_{\infty ; \Sigma},\|b\|_{\infty ; \Sigma},\|c\|_{\infty},|d|_{1+\beta, \frac{1+\beta}{2} ; \bar{Q}}\right)$. We infer from the choice of $\theta$ that $2 \nabla \theta \cdot \nabla \hat{q}+(\Delta \theta) \hat{q} \in C^{2+\beta, 1+\frac{\beta}{2}}(\bar{Q})$, and hence that $v_{3}$ lies in $C^{\beta, \frac{\beta}{2}}(\bar{Q})$, and one can estimate

$$
\left|v_{3}\right|_{\beta, \frac{\beta}{2} ; \bar{Q}} \leq C\left(\Omega, \omega, \mathcal{O}, T,\|a\|_{\infty ; \Sigma},\|b\|_{\infty ; \Sigma},\|c\|_{\infty},|d|_{1+\beta, \frac{1+\beta}{2} ; \bar{Q}}\right)\left(\|\xi\|_{\infty}+\|\hat{v}\|_{L^{2}}\right) .
$$

In view of the previous considerations on each term $v_{i}, 1 \leq i \leq 3$, and using estimate (2.8), one concludes that $v$ given by $(2.14)$ lies in $C^{\beta, \frac{\beta}{2}}(\bar{Q})$ and that (2.9) holds. Finally, it is an easy exercise to see that $(y, q)$ defined by $(2.13)$ and $(2.12)$, together with this control function $v$, solves (2.1), (2.2), and (1.7). The only delicate point could be to check that $y(x, 0)=0$ in $\Omega$. But this follows immediately from the interior regularity of $\hat{q}$ (which, in particular, gives $\hat{q} \in C\left([0, T] ; H^{1}(B \backslash\right.$ $\left.\left.\bar{B}_{2}\right)\right)$ ), the choice of $\theta$, and the fact that $\hat{q}(x, 0)=0$ in $\Omega$. This ends the proof of Proposition 2.3.

We end this section by giving the proof of Lemma 2.6 , which relies on a localization argument.

Proof of Lemma 2.6. Assume the hypothesis in the statement, with $\beta \in(0,1)$ and $\mathcal{V}$ being fixed. Given an open set $\mathcal{V}^{\prime} \subset \subset \mathcal{V}$, we consider a regular open set $\mathcal{V}_{1}$, with $\mathcal{V}^{\prime} \subset \subset \mathcal{V}_{1} \subset \subset \mathcal{V}$. According to the regularity of $h$ and the potential $\widetilde{a}$, we can apply the second point of Lemma 2.4, with $r=(N+2) /(1-\beta)$ (thus $\gamma=r$, since $r>N+2)$, and deduce that $u, \frac{\partial u}{\partial x_{i}} \in X^{r}\left(0, T ; \mathcal{V}_{1}\right), i=1, \ldots, N$, together with the estimate

$$
\|u\|_{X^{r}\left(0, T ; \mathcal{V}_{1}\right)}+\|\nabla u\|_{X^{r}\left(0, T ; \mathcal{V}_{1}\right)^{N}} \leq C\left[\|h\|_{L^{r}\left(W^{1, r}(\mathcal{V})\right)}+\|u\|_{L^{2}\left(H^{1}\right) \cap C\left(L^{2}\right)}\right],
$$

with $C>0$ depending on $\Omega, \mathcal{V}, \mathcal{V}^{\prime}, T,\|\widetilde{a}\|_{\infty}$ and $\|\nabla \widetilde{a}\|_{L^{r}}$. Since $r>N+2$, by Lemma 2.5 we get

$$
u, \frac{\partial u}{\partial x_{i}} \in C^{1+\beta, \frac{1+\beta}{2}}\left(\overline{\mathcal{V}}_{1} \times[0, T]\right), \quad i=1, \ldots, N,
$$

with

$$
\begin{gathered}
|u|_{1+\beta, \frac{1+\beta}{2} ; \overline{\mathcal{V}}_{1} \times[0, T]}+|\nabla u|_{1+\beta, \frac{1+\beta}{2} ; \overline{\mathcal{V}}_{1 \times[0, T]}} \\
\leq C\left(\Omega, \mathcal{V}, \mathcal{V}^{\prime}, T,\|\widetilde{a}\|_{\infty},\|\nabla \widetilde{a}\|_{L^{r}}\right)\left(\|h\|_{L^{r}\left(W^{1, r}(\mathcal{V})\right)}+\|u\|_{L^{2}\left(H^{1}\right) \cap C\left(L^{2}\right)}\right)
\end{gathered} .
$$

We claim that, indeed, $u$ lies in $C^{3+\beta, \frac{3+\beta}{2}}\left(\overline{\mathcal{V}}^{\prime} \times[0, T]\right)$ and satisfies (2.18). To this end, let $\zeta \in \mathcal{D}\left(\mathcal{V}_{1}\right)$ be such that $\zeta \equiv 1$ in $\mathcal{V}^{\prime}$ and set $w=\zeta u$. Then $w$ solves

$$
\begin{cases}\partial_{t} w-\Delta w=\widetilde{h} & \text { in } Q, \\ w=0 \quad \text { on } \Sigma, & w(x, 0)=0 \quad \text { in } \Omega,\end{cases}
$$

with $\widetilde{h}=\zeta h-[\widetilde{a} \zeta u+2 \nabla \zeta \cdot \nabla u+(\Delta \zeta) u]$. The regularity of $h$ and $\widetilde{a}$, together with (2.19), gives $\widetilde{h} \in C^{1+\beta, \frac{1+\beta}{2}}(\bar{Q})$, and using (2.20), one has

$$
|\widetilde{h}|_{1+\beta, \frac{1+\beta}{2} ; \bar{Q}} \leq C\left(|h|_{1+\beta, \frac{1+\beta}{2} ; \overline{\mathcal{V}} \times[0, T]}+\|u\|_{L^{2}\left(H^{1}\right) \cap C\left(L^{2}\right)}\right),
$$


with $C=C\left(\Omega, \mathcal{V}, \mathcal{V}^{\prime}, T,|\widetilde{a}|_{1+\beta, \frac{1+\beta}{2} ; \bar{Q}}\right)$. Since $\partial \Omega \in C^{3+\beta}$ and the compatibility condition of order 1 for system (2.21) is trivially fulfilled, one can apply Theorem 5.2 of [9] to obtain $w \in C^{3+\beta, \frac{3+\beta}{2}}(\bar{Q})$, with

$$
|w|_{3+\beta, \frac{3+\beta}{2} ; \bar{Q}} \leq C(\Omega, T)|\widetilde{h}|_{1+\beta, \frac{1+\beta}{2} ; \bar{Q}} .
$$

Finally, recalling that $u \equiv w$ in $\mathcal{V}^{\prime}$, one infers the desired interior regularity of $u$, and estimate (2.18) holds, using (2.23) and (2.22).

3. Proof of Theorem 1.2. We begin this section by recalling the following result for linear systems of the form

$$
\left\{\begin{array}{l}
\partial_{t} u-\Delta u+c u=h \quad \text { in } Q \\
\partial_{n} u+a u=0 \quad \text { on } \Sigma \\
u(x, 0)=u_{0}(x) \text { in } \Omega
\end{array}\right.
$$

whose proof is given in [9, Theorem 5.3, p. 320].

Lemma 3.1. Assume that $\partial \Omega \in C^{2+\beta}$ for some $\beta \in(0,1)$. Let $a \in C^{1+\beta, \frac{1+\beta}{2}}(\bar{\Sigma})$ and $c \in C^{\beta, \frac{\beta}{2}}(\bar{Q})$ be given. Then, for any $h \in C^{\beta, \frac{\beta}{2}}(\bar{Q})$ and $u_{0} \in C^{2+\beta}(\bar{\Omega})$ satisfying the compatibility condition

$$
\partial_{n} u_{0}(x)+a(x, 0) u_{0}(x)=0 \quad \text { on } \partial \Omega,
$$

system (3.1) admits exactly one solution $u \in C^{2+\beta, 1+\frac{\beta}{2}}(\bar{Q})$ verifying the estimate

$$
|u|_{2+\beta, 1+\frac{\beta}{2} ; \bar{Q}} \leq C\left(\Omega, T,|a|_{1+\beta, \frac{1+\beta}{2} ; \bar{\Sigma}},|c|_{\beta, \frac{\beta}{2} ; \bar{Q}}\right)\left(|h|_{\beta, \frac{\beta}{2} ; \bar{Q}}+\left|u_{0}\right|_{2+\beta ; \bar{\Omega}}\right) .
$$

Let us now prove Theorem 1.2. Assume the hypothesis in the statement. From the considerations in the first section, the proof falls naturally into two steps.

Step 1. Existence of a regular control solving the nonlinear null controllability problem (1.5)-(1.7): The fixed point argument. Let $G$ and $g$ be the $C^{2}$ functions defined by

$$
G(s)=\left\{\begin{array}{ll}
\frac{F(s)}{s} & \text { if } s \neq 0, \\
F^{\prime}(0) & \text { if } s=0,
\end{array} \quad g(s)= \begin{cases}\frac{f(s)}{s} & \text { if } s \neq 0 \\
f^{\prime}(0) & \text { if } s=0 .\end{cases}\right.
$$

Let us set

$$
Z=C^{1}(\bar{Q}) \cap C^{1+\beta, \frac{1+\beta}{2}}(\bar{Q}) .
$$

For fixed $z \in \bar{B}(0 ; 1) \subset Z$, we consider the linear systems

$$
\begin{aligned}
& \left\{\begin{array}{l}
\partial_{t} y-\Delta y+G(z) y=\xi+v \mathbf{1}_{\omega} \text { in } Q \\
\partial_{n} y+g(z) y=0 \text { on } \Sigma, \\
y(x, 0)=0 \text { in } \Omega
\end{array}\right. \\
& \left\{\begin{array}{l}
-\partial_{t} q-\Delta q+F^{\prime}(z) q=y \mathbf{1}_{\mathcal{O}} \text { in } Q, \\
\partial_{n} q+f^{\prime}(z) q=0 \text { on } \Sigma \\
q(x, T)=0 \text { in } \Omega,
\end{array}\right.
\end{aligned}
$$


with potentials $G(z), F^{\prime}(z) \in Z$ and $g(z), f^{\prime}(z) \in \widetilde{Z}=C^{1}(\bar{\Sigma}) \cap C^{1+\beta, \frac{1+\beta}{2}}(\bar{\Sigma})$. By abuse of notation, from now on we will let $g(z)$ (resp., $f^{\prime}(z)$ ) stand for both the function $g(z)$ in $Z$ and its restriction to $\bar{\Sigma}$ (resp., for both $f^{\prime}(z) \in Z$ and its restriction to $\bar{\Sigma}$ ). Let us set

$$
\mathcal{M}(\Omega, \omega, \mathcal{O}, T, F, f)=\sup _{z \in \bar{B}(0 ; 1)} M_{z}
$$

where $M_{z}$ is, for fixed $z \in \bar{B}(0 ; 1)$, the positive constant (depending on $\Omega, \omega, \mathcal{O}, T$, $\|g(z)\|_{Z},\left\|f^{\prime}(z)\right\|_{Z},\|G(z)\|_{Z}$, and $\left.\left\|F^{\prime}(z)\right\|_{Z}\right)$ provided by Proposition 2.1. Let $\xi$ in $C^{\beta, \frac{\beta}{2}}(\bar{Q})$ satisfy (1.4), with $\eta>0$ to be chosen later (hence also verifying (2.7) with $M=M_{z}$ for all $\left.z \in \bar{B}(0 ; 1)\right)$. From Proposition 2.3 , there exists $v_{z} \in C^{\beta, \frac{\beta}{2}}(\bar{Q})$ such that the associated solution $\left(y_{z}, q_{z}\right)$ of $(3.3),(3.4)$ lies in $C^{2+\beta, 1+\frac{\beta}{2}}(\bar{Q}) \times C^{2+\beta, 1+\frac{\beta}{2}}(\bar{Q})$ (by Lemma 3.1) and satisfies (1.7). Moreover, one has

$$
\left|v_{z}\right|_{\beta, \frac{\beta}{2} ; \bar{Q}} \leq C(\Omega, \omega, \mathcal{O}, T, z)\left(|\xi|_{\beta, \frac{\beta}{2} ; \bar{Q}}+\left\|\exp \left(\frac{M_{z}}{2 t}\right) \xi\right\|_{L^{2}}\right)
$$

with $C(\Omega, \omega, \mathcal{O}, T, z)=C\left(\Omega, \omega, \mathcal{O}, T,\|g(z)\|_{Z},\left\|f^{\prime}(z)\right\|_{Z},\|G(z)\|_{Z},\left\|F^{\prime}(z)\right\|_{Z}\right)$, and $y_{z}$ satisfies an estimate such as (3.2); hence

$$
\left|y_{z}\right|_{2+\beta, 1+\frac{\beta}{2} ; \bar{Q}} \leq C_{1}(\Omega, \omega, \mathcal{O}, T, z)\left(|\xi|_{\beta, \frac{\beta}{2} ; \bar{Q}}+\left\|\exp \left(\frac{M_{z}}{2 t}\right) \xi\right\|_{L^{2}}\right),
$$

with $C_{1}(\Omega, \omega, \mathcal{O}, T, z)=C_{1}\left(\Omega, \omega, \mathcal{O}, T,\|g(z)\|_{Z},\left\|\left.f^{\prime}(z)\right|_{Z},\right\| G(z)\left\|_{Z},\right\| F^{\prime}(z) \|_{Z}\right)$ (and a similar estimate for $q_{z}$ holds). Then, for any $z \in \bar{B}(0 ; 1)$ one has

$$
\begin{aligned}
& \left|v_{z}\right|_{\beta, \frac{\beta}{2} ; \bar{Q}} \leq \widetilde{C}(\Omega, \omega, \mathcal{O}, T, F, f)\left(|\xi|_{\beta, \frac{\beta}{2} ; \bar{Q}}+\left\|\exp \left(\frac{\mathcal{M}}{2 t}\right) \xi\right\|_{L^{2}}\right), \\
& \left|y_{z}\right|_{2+\beta, 1+\frac{\beta}{2} ; \bar{Q}} \leq C_{2}(\Omega, \omega, \mathcal{O}, T, F, f)\left(|\xi|_{\beta, \frac{\beta}{2} ; \bar{Q}}+\left\|\exp \left(\frac{\mathcal{M}}{2 t}\right) \xi\right\|_{L^{2}}\right),
\end{aligned}
$$

together with a similar estimate for $q_{z}$, with

$$
\begin{aligned}
\widetilde{C}(\Omega, \omega, \mathcal{O}, T, F, f) & =\sup _{z \in \bar{B}(0 ; 1)} C(\Omega, \omega, \mathcal{O}, T, z), \\
C_{2}(\Omega, \omega, \mathcal{O}, T, F, f) & =\sup _{z \in \bar{B}(0 ; 1)} C_{1}(\Omega, \omega, \mathcal{O}, T, z) .
\end{aligned}
$$

For each $z \in \bar{B}(0 ; 1) \subset Z$, we consider the families

$$
\begin{gathered}
\mathcal{A}(z)=\left\{v \in C^{\beta, \frac{\beta}{2}}(\bar{Q}):(y, q) \text { satisfies }(3.3),(3.4), \text { and }(1.7), v \text { verifying }(3.6)\right\}, \\
\Lambda(z)=\{y:(y, q) \text { solves }(3.3),(3.4) \text { with } v \in \mathcal{A}(z)\} .
\end{gathered}
$$

One can then define the set-valued mapping $\Lambda: z \in \bar{B}(0 ; 1) \subset Z \mapsto \Lambda(z) \subset Z$. For fixed $z \in \bar{B}(0 ; 1)$, each $y \in \Lambda(z)$ lies in $C^{2+\beta, 1+\frac{\beta}{2}}(\bar{Q})$ and satisfies (3.7), and thus

$$
\|y\|_{Z} \leq C_{2}(\Omega, \omega, \mathcal{O}, T, F, f)\left(|\xi|_{\beta, \frac{\beta}{2} ; \bar{Q}}+\left\|\exp \left(\frac{\mathcal{M}}{2 t}\right) \xi\right\|_{L^{2}}\right) \text {. }
$$


We claim that there exists $\eta(\Omega, \omega, \mathcal{O}, T, F, f)>0$ such that if a source term $\xi \in C^{\beta, \frac{\beta}{2}}(\bar{Q})$ satisfies (1.4), with $\mathcal{M}$ given by (3.5), then the Kakutani fixed point theorem can be applied to $\Lambda$. First, for fixed $z \in \bar{B}(0 ; 1) \subset Z$, it is easy to check that $\Lambda(z)$ is a nonempty closed convex subset of $Z$ (here we use the linear character of systems (3.3) and (3.4)). By estimate (3.7), $\Lambda(z)$ is a bounded set in $C^{2+\beta, 1+\frac{\beta}{2}}(\bar{Q})$. Since this space is compactly embedded into $Z$, one infers that each $\Lambda(z)$ is a compact subset of $Z$. Furthermore, there exists a fixed compact set $K \subset Z$ such that $\Lambda(z) \subset K$ for all $z \in \bar{B}(0 ; 1)$.

In the second place, $\Lambda$ is proved to be an upper hemicontinuous multivalued mapping, or, equivalently, it is proved that for any bounded linear form $\mu \in Z^{\prime}$, the function

$$
z \in \bar{B}(0 ; 1) \subset Z \mapsto \sup _{y \in \Lambda(z)}\langle\mu, y\rangle \in \mathbb{R}
$$

is upper semicontinuous. To this end, it suffices to show that the set

$$
B_{\lambda, \mu}=\left\{z \in \bar{B}(0 ; 1): \sup _{y \in \Lambda(z)}\langle\mu, y\rangle \geq \lambda\right\}
$$

is closed in $Z$ for any $\lambda \in \mathbb{R}$ and any $\mu \in Z^{\prime}$. Let us fix $\lambda \in \mathbb{R}$ and $\mu \in Z^{\prime}$, and consider a sequence $\left\{z_{n}\right\}_{n \geq 1} \subset B_{\lambda, \mu}$ such that

$$
z_{n} \rightarrow z \text { in } Z \text {. }
$$

Our aim is to see that $z \in B_{\lambda, \mu}$. As stated above, each $\Lambda\left(z_{n}\right)$ is a compact set in $Z$. Then for fixed $n \geq 1$ one has

$$
\sup _{y \in \Lambda\left(z_{n}\right)}\langle\mu, y\rangle=\left\langle\mu, y_{n}\right\rangle \geq \lambda
$$

for some $y_{n} \in \Lambda\left(z_{n}\right)$. By the definition of $\Lambda\left(z_{n}\right)$ and $\mathcal{A}\left(z_{n}\right)$, there exist $v_{n} \in C^{\beta, \frac{\beta}{2}}(\bar{Q})$ satisfying

$$
\left|v_{n}\right|_{\beta, \frac{\beta}{2} ; \bar{Q}} \leq \widetilde{C}(\Omega, \omega, \mathcal{O}, T, F, f)\left(|\xi|_{\beta, \frac{\beta}{2} ; \bar{Q}}+\left\|\exp \left(\frac{\mathcal{M}}{2 t}\right) \xi\right\|_{L^{2}}\right)
$$

$(\widetilde{C}(\Omega, \omega, \mathcal{O}, T, F, f)$ as in $(3.6))$ and $q_{n} \in C^{2+\beta, 1+\frac{\beta}{2}}(\bar{Q})$ such that $\left(y_{n}, q_{n}\right)$ together with $v_{n}$ solve

$$
\begin{aligned}
& \left\{\begin{array}{l}
\partial_{t} y_{n}-\Delta y_{n}+G\left(z_{n}\right) y_{n}=\xi+v_{n} \mathbf{1}_{\omega} \text { in } Q, \\
\partial_{n} y_{n}+g\left(z_{n}\right) y_{n}=0 \text { on } \Sigma, \\
y_{n}(x, 0)=0 \text { in } \Omega,
\end{array}\right. \\
& \left\{\begin{array}{l}
-\partial_{t} q_{n}-\Delta q_{n}+F^{\prime}\left(z_{n}\right) q_{n}=y_{n} \mathbf{1}_{\mathcal{O}} \text { in } Q, \\
\partial_{n} q_{n}+f^{\prime}\left(z_{n}\right) q_{n}=0 \text { on } \Sigma, \\
q_{n}(x, T)=0, \quad q_{n}(x, 0)=0 \text { in } \Omega .
\end{array}\right.
\end{aligned}
$$

From (3.11) and (3.7), $\left\{v_{n}\right\}$ and $\left\{\left(y_{n}, q_{n}\right)\right\}$ are uniformly bounded in $C^{\beta, \frac{\beta}{2}}(\bar{Q})$ and $C^{2+\beta, 1+\frac{\beta}{2}}(\bar{Q}) \times C^{2+\beta, 1+\frac{\beta}{2}}(\bar{Q})$, respectively. Taking into account the compact embed$\operatorname{ding}$ of $C^{\beta, \frac{\beta}{2}}(\bar{Q})$ (resp., $C^{2+\beta, 1+\frac{\beta}{2}}(\bar{Q})$ ) into $C^{0}(\bar{Q})$ (resp., $Z$ ), there exist subsequences (still denoted by $\left\{v_{n}\right\}$ and $\left.\left\{\left(y_{n}, q_{n}\right)\right\}\right)$ such that

$$
v_{n} \rightarrow \widetilde{v} \quad \text { in } C^{0}(\bar{Q}), \quad\left(y_{n}, q_{n}\right) \rightarrow(\widetilde{y}, \widetilde{q}) \quad \text { in } Z \times Z
$$


for some $\widetilde{v} \in C^{0}(\bar{Q}),(\widetilde{y}, \widetilde{q}) \in Z \times Z$. It is easily seen that, in fact, $\widetilde{v} \in C^{\beta, \frac{\beta}{2}}(\bar{Q})$. On account of the regularity of $F$ and $f$, from (3.9) one also has

$$
\begin{gathered}
G\left(z_{n}\right) \rightarrow G(z) \quad \text { and } \quad F^{\prime}\left(z_{n}\right) \rightarrow F^{\prime}(z) \quad \text { in } Z, \\
g\left(z_{n}\right) \rightarrow g(z) \quad \text { and } \quad f^{\prime}\left(z_{n}\right) \rightarrow f^{\prime}(z) \quad \text { in } \widetilde{Z} .
\end{gathered}
$$

We can then pass to the limit in (3.11)-(3.13) and deduce that $(\widetilde{y}, \widetilde{q})$ solves (3.3), (3.4), and (1.7) with control term $\widetilde{v} \in \mathcal{A}(z)$. Thus, $\widetilde{y} \in \Lambda(z)$, and taking limits in (3.10), one infers that

$$
\sup _{y \in \Lambda(z)}\langle\mu, y\rangle \geq\langle\mu, \widetilde{y}\rangle \geq \lambda .
$$

We conclude that $z \in B_{\lambda, \mu}$; hence, $\Lambda$ is an upper hemicontinuous mapping.

Now let $\eta=\eta(\Omega, \omega, \mathcal{O}, T, F, f)>0$ be such that $\eta \leq C_{2}(\Omega, \omega, \mathcal{O}, T, F, f)^{-1}$. Then, for a given source term $\xi \in C^{\beta, \frac{\beta}{2}}(\bar{Q})$ satisfying (1.4), with $\mathcal{M}$ given by (3.5), we infer from (3.8) that any $y \in \Lambda(\bar{B}(0 ; 1))$ verifies $\|y\|_{Z} \leq 1$; that is, $\Lambda$ maps the nonempty closed convex set $\bar{B}(0 ; 1)$ into itself. We can then apply the Kakutani fixed point theorem and conclude that there exists $\bar{y} \in Z$ such that $\bar{y} \in \Lambda(\bar{y})$. Hence, there exists $v \in C^{\beta, \frac{\beta}{2}}(\bar{Q})$, solving the nonlinear null controllability problem (1.5)-(1.7) (for $\left.y_{0}=0\right)$. Moreover, by (3.6) one can estimate

$$
|v|_{\beta, \frac{\beta}{2} ; \bar{Q}} \leq \widetilde{C}(\Omega, \omega, \mathcal{O}, T, F, f) \eta .
$$

Step 2. Existence of a control insensitizing the functional $\Phi$. Let us see that there exists $\eta(\Omega, \omega, \mathcal{O}, T, F, f)>0$ such that for any $\xi \in C^{\beta, \frac{\beta}{2}}(\bar{Q})$ satisfying (1.4), with $\mathcal{M}$ given by (3.5), the control $v$ in the previous step can be chosen so that, for $\tau$ small enough, the existence of a solution of (1.1) (with $y_{0}=0$ ) in $C^{2+\beta, 1+\frac{\beta}{2}}(\bar{Q})$ is ensured. This will conclude the proof of the theorem, since such a control $v$ will then insensitize the functional $\Phi$ given by (1.2), in view of Proposition 1.3.

We use the following result, which can be proved by linearizing and applying an appropriate fixed point argument.

Lemma 3.2. Assume that $\partial \Omega \in C^{2+\beta}$ for some $\beta \in(0,1)$. Let $F \in C^{2}(\mathbb{R})$ and $f \in C^{3}(\mathbb{R})$ be given. Then, there exists $\delta>0$ (depending on $\Omega, T, F$, and $f$ ) with the property that for any $h \in C^{\beta, \frac{\beta}{2}}(\bar{Q})$ and $u_{0} \in C^{2+\beta}(\bar{\Omega})$ satisfying

$$
|h-F(0)|_{\beta, \frac{\beta}{2} ; \bar{Q}}+|f(0)|+\left|u_{0}\right|_{2+\beta ; \bar{\Omega}} \leq \delta
$$

and the compatibility condition

$$
\partial_{n} u_{0}+f\left(u_{0}\right)=0 \quad \text { on } \partial \Omega
$$

the nonlinear system

$$
\left\{\begin{array}{l}
\partial_{t} u-\Delta u+F(u)=h \quad \text { in } Q \\
\partial_{n} u+f(u)=0 \quad \text { on } \Sigma \\
u(x, 0)=u_{0}(x) \quad \text { in } \Omega
\end{array}\right.
$$

admits a unique solution $u \in C^{2+\beta, 1+\frac{\beta}{2}}(\bar{Q})$.

Let us consider $X=C^{2+\beta}(\bar{\Omega}) \cap H_{0}^{2}(\Omega)$, with $\beta \in(0,1)$ as in the statement. Let $\delta>0$ be provided by Lemma 3.2 and let $\mathcal{M}(\Omega, \omega, \mathcal{O}, T, F, f)$ be given by (3.5). 
Recalling (3.14), one can choose $\eta=\eta(\Omega, \omega, \mathcal{O}, T, F, f)>0$ small enough so that for any $\xi \in C^{\beta, \frac{\beta}{2}}(\bar{Q})$ verifying (1.4), $\hat{y}_{0} \in X$ with $\left\|\hat{y}_{0}\right\|_{X}=1$, and $\tau \in \mathbb{R}$ small enough, one has

$$
\left|\xi+v \mathbf{1}_{\omega}\right|_{\beta, \frac{\beta}{2} ; \bar{Q}}+\left|\tau \hat{y}_{0}\right|_{2+\beta ; \bar{\Omega}} \leq \delta .
$$

Since the initial datum $\tau \hat{y}_{0}$ satisfies (3.15) (by choice of $X$ ), one infers from Lemma 3.2 that system (1.1) possesses a solution $y(\cdot, \cdot ; \tau, v) \in C^{2+\beta, 1+\frac{\beta}{2}}(\bar{Q})$, which ends the proof of Theorem 1.2.

\section{REFERENCES}

[1] O. Bodart And C. FABRE, Controls insensitizing the norm of the solution of a semilinear heat equation, J. Math. Anal. Appl., 195 (1995), pp. 658-683.

[2] O. Bodart, M. González-Burgos, and R. PÉrez-García, Insensitizing controls for a semilinear heat equation with a superlinear nonlinearity, C. R. Acad. Sci. Paris, Sér. I Math., 335 (2002), pp. 677-682.

[3] O. Bodart, M. González-Burgos, and R. PÉrez-García, Existence of insensitizing controls for a semilinear heat equation with a superlinear nonlinearity, Comm. Partial Differential Equations, to appear.

[4] O. Bodart, M. González-Burgos, and R. PÉRez-García, Insensitizing controls for a heat equation with a nonlinear term involving the state and the gradient, Nonlinear Anal., 57 (2004), pp. 687-711.

[5] A. Doubova, E. Fernández-Cara, and M. González-Burgos, On the controllability of the heat equation with nonlinear boundary Fourier conditions, J. Differential Equations, 196 (2004), pp. 385-417.

[6] C. Fabre, J.-P. Puel, and E. Zuazua, Approximate controllability of the semilinear heat equation, Proc. Roy. Soc. Edinburgh Sect. A, 125 (1995), pp. 36-61.

[7] E. Fernández-CARA AND E. ZuAZuA, Null and approximate controllability for weakly blowing up semilinear heat equations, Ann. Inst. H. Poincaré Anal. Non Linéaire, 17 (2000), pp. 583-616.

[8] A. Fursikov and O. Yu. Imanuvilov, Controllability of Evolution Equations, Lecture Notes Ser. 34, Seoul National University, Seoul, Korea, 1996.

[9] O. A. Ladyzenskaya, V. A. Solonnikov, and N. N. Uraltzeva, Linear and Quasilinear Equations of Parabolic Type, Transl. Math. Monogr. 23, AMS, Providence, RI, 1967.

[10] J.-L. Lions, Quelques notions dans l'analyse et le contrôle de systèmes à données incomplètes, in Proceedings of the XIth Congress on Differential Equations and Applications/First Congress on Applied Mathematics, University of Málaga, Málaga, Spain, 1990, pp. 43-54.

[11] L. De Teresa, Insensitizing controls for a semilinear heat equation, Comm. Partial Differential Equations, 25 (2000), pp. 39-72.

[12] E. ZuAzUA, Exact boundary controllability for the semilinear wave equation, in Nonlinear Partial Differential Equations and Their Applications, Vol. X, Pitman Res. Notes Math. Ser. 220, H. Brezis and J.-L. Lions, eds., Longman Sci. Tech., Harlow, UK, 1991, pp. 357-391. 